\title{
A highly sensitive, large area, and self-powered UV photodetector based on coalesced gallium nitride nanorods/graphene/silicon (111) heterostructure
}

\author{
Cite as: Appl. Phys. Lett. 117, 191103 (2020); doi: 10.1063/5.0018076 \\ Submitted: 12 June 2020 • Accepted: 2 November 2020 • \\ Published Online: 13 November 2020

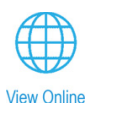 \\ Nur 'Adnin Akmar Zulkifli,, ${ }^{1,2}$ Kwangwook Park, ${ }^{3,4}$ iD Jung-Wook Min, ${ }^{5}$ Boon S. Ooi, ${ }^{5}$ iD Rozalina Zakaria, ${ }^{2}$ iD \\ Jongmin Kim, ${ }^{6, a)}$ and Chee Leong $\operatorname{Tan}^{1,2, a)}$ (iD \\ AFFILIATIONS \\ ${ }^{7}$ School of Electronic and Optical Engineering and College of Microelectronics, Nanjing University of Posts and \\ Telecommunications, Nanjing 210023, China \\ ${ }^{2}$ Photonic Research Centre, University of Malaya, 50603 Kuala Lumpur, Malaysia \\ ${ }^{3}$ Division of Advanced Materials Engineering, Jeonbuk National University, Jeonju 54896, South Korea \\ ${ }^{4}$ Hydrogen and Fuel Cell Center, Jeonbuk National University, Jeonju 54896, South Korea \\ ${ }^{5}$ Photonics Laboratory, King Abdullah University of Science and Technology, Thuwal 23955, Saudi Arabia \\ ${ }^{6}$ Korea Advanced Nano Fab Center, Suwon 16229, South Korea
}

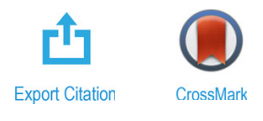

a) Authors to whom correspondence should be addressed: jongmin.kim@kanc.re.kr and cheelong@njupt.edu.cn

\begin{abstract}
In this paper, we demonstrate an ultraviolet photodetector (UV-PD) that uses coalesced gallium nitride (GaN) nanorods (NRs) on a graphene/Si (111) substrate grown by plasma-assisted molecular beam epitaxy. We report a highly sensitive, self-powered, and hybrid GaN $\mathrm{NR} /$ graphene/Si (111) PD with a relatively large $100 \mathrm{~mm}^{2}$ active area, a high responsivity of $17.4 \mathrm{~A} / \mathrm{W}$, a high specific detectivity of $1.23 \times 10^{13}$ Jones, and fast response speeds of $13.2 / 13.7 \mu \mathrm{s}(20 \mathrm{kHz})$ under a UV light of $355 \mathrm{~nm}$ at zero bias voltage. The results show that the thin graphene acts as a perfect interface for GaN NRs, encouraging growth with minimum defects on the Si substrate. Our results suggest that the GaN NR/graphene/Si (111) heterojunction has a range of interesting properties that make it well-suited for a variety of photodetection applications.
\end{abstract}

Published under license by AIP Publishing. https://doi.org/10.1063/5.0018076

Nitride-based compound semiconductors are promising materials with a wide range of potential optoelectronic applications, as their wide tunable direct bandgap allows for the fabrication of highperformance emission and sensing devices in the ultra-violet, visible, and infrared spectral range. ${ }^{1,2}$ Gallium nitride $(\mathrm{GaN})$, with a bandgap of $3.4 \mathrm{eV}$, is widely understood to be a potentially useful addition to high-power and high-temperature semiconductor devices, specifically those employing ultraviolet (UV) detection. ${ }^{3-6}$ Unfortunately, high defect density, short carrier lifetime, and low carrier mobility inherent to traditional GaN-based photodetectors (PDs) lead to low photocarrier collection. GaN nanorods (NRs), due to their high photoconduction and better sensitivity toward light, can overcome the issues associated with three-dimensional (3D) planar GaN layers and, in recent decades, have become attractive potential components in a wide range of optoelectronic devices, including as electrical pump lasers,
PDs, and solar cells. ${ }^{7}$ PDs with one-dimensional (1D) NRs have demonstrated excellent performance, manifesting high responsivity and detectivity, ${ }^{8-10}$ as a result of various inherent properties: large surface-to-volume ratios, light scattering (between the NRs), and reduced cross section to the quantum confinement size regime. ${ }^{11}$ In these PDs, electrons are generally confined to the core, as opposed to the surface, due to the high potential barrier at the NRs' surface. Cross-section reduction from a large (3D) to small (1D) structure significantly reduces the scattering in $1 \mathrm{D}$ structures, thereby effectively increasing overall electron mobility and minimizing charge transit time. ${ }^{12}$ Photocarrier lifetime is also prolonged as a result of the presence of surface trap states in the NRs. ${ }^{13}$ Group-III nitride NRs have been widely grown on bulk crystalline substrates such as silicon ${ }^{14,15}$ and sapphire, ${ }^{16,17}$ resulting in NRs with a high-quality epitaxial relationship with their substrate. 
Despite these advantages, deficiencies in the bulk substrates (low electrical or thermal conductivities, opacity, weight, and rigidity) are responsible for ultimately disappointing device performance. Accordingly, there has been an extensive search for replacement substrates that will not impair the quality of NRs. ${ }^{18}$ One method that has shown promise is to grow or transfer two-dimensional (2D) atomic layer materials to a carrier substrate as a seed layer for NR growth, a process referred to as heteroepitaxy. This allows for a more comprehensive range of materials and a potentially better fit between host carrier characteristics and the device requirements. There is also a unique feature of graphene that it forms a Schottky junction with the nanostructures of other semiconducting materials, including $\mathrm{Si}, \mathrm{GaN}$, and $\mathrm{MoS}_{2}$, which makes it a favorable candidate for achieving outstanding device performance. ${ }^{19}$ Accordingly, $2 \mathrm{D}$ graphene has been proposed as a substrate to grow III-V semiconductor NRs. ${ }^{20}$ Its remarkable characteristics, including its high carrier mobility, ${ }^{21}$ broad spectral absorption wavelength, and fast carrier transport, have attracted numerous researchers interested in building hybrid devices. ${ }^{22-24}$ Prior researchers have used graphene as a transparent contact with GaN NRs due to its high conductivity and low optical absorbance (approximately $2.3 \%$ ) for light in a wavelength range of $300 \mathrm{~nm}$ to the nearinfrared, ${ }^{25}$ and produced around $25 \mathrm{~A} / \mathrm{W}$ responsivity as a result. ${ }^{26}$ Others have compared photocurrent production and responsivity in GaN PDs with and without graphene, determining that a GaN PD with graphene produces $35 \times$ more photocurrent and higher responsivity than it does without graphene. ${ }^{27}$

In our device, graphene acts as an efficient carrier transport channel between GaN NRs and Si (111), significantly increasing the charge collection efficiency. Graphene also allows GaN NRs to grow on the $\mathrm{Si}$ substrate without any growth constraints, such as lattice mismatch or growth defects. The growth of GaN NRs on a graphene/foreign substrate is linked to the fabrication method used. Growing on heterogeneous substrates through plasma-assisted molecular beam epitaxy (PA-MBE), GaN NRs follow a self-assembled growth mechanism that has the inhomogeneity of the NR morphology. ${ }^{28}$ Additionally, a low filling factor of NRs can cause infiltration during the device fabrication process during the contact metal deposition step.

Accordingly, the addition of a planarization step that uses polyimide $^{29}$ or parylene $\mathrm{e}^{30}$ is essential to achieving robust NR-based devices. This step, which involves polymer deposition, curing, and surface etching, results in an unfortunate increase in fabrication cost and time. It may also result in deteriorated device performance, as there are only a limited number of UV-transparent and high-temperature tolerant polymer materials suitable for heat dissipation. To overcome these obstacles, we intentionally induced coalesced GaN NRs along the $c$ plane orientation with a fill factor of greater than $95 \%,{ }^{31}$ and directly deposited contact metal on GaN NRs for a large active area of $1 \mathrm{~cm}^{2}$ without any planarization step.

In this paper, we report the fabrication of a large area coalesced GaN NR/graphene/Si (111) PD of $100 \mathrm{~mm}^{2}$. The use of Si as a carrier substrate also provides a double Schottky junction to GaN NR/ graphene, as it adds a photon absorption layer, leading to a highly sensitive UV PD with high speed and responsivity. We report a selfpowered UV PD, which is highly sensitive to the UV light of $355 \mathrm{~nm}$. At a $48 \mu \mathrm{W} / \mathrm{cm}^{2}$ power light intensity, it has a high responsivity of 17.4 A/W, a specific detectivity of $1.23 \times 10^{13}$ Jones, a current on/off ratio of nearly $10^{5}$, and a fast response speed of $\sim 13 \mu$ s at zero bias voltage. The fabricated GaN NR/graphene/Si (111) UV PD is an asymmetrical metal-semiconductor-metal (MSM) PD, which consists of two different Schottky junctions Au/GaN NRs and the Au/Si (111) substrate. The thin graphene acts as an interface for GaN NRs to grow on a $\mathrm{Si}$ substrate with minimum defects. The dark current clearly shows that the graphene has no bandgap as it acts as the carrier transport channel, which allows electron and hole transfer between the GaN NRs and the Si substrate. Our results show outstanding overall performance compared to previously reported GaN-based PDs, and this high-functioning GaN NR/graphene/Si (111) PD shows promise for future applications.

For sample growth, graphene was deposited on $\mathrm{Cu}$-foil using atmospheric chemical vapor deposition (APCVD). To deposit graphene, the quartz tube was heated to a temperature of approximately $1000^{\circ} \mathrm{C}$ under a $\mathrm{H}_{2} / \mathrm{Ar}$ atmosphere. Graphene was grown with 20 sccm of $\mathrm{CH}_{4}$ for $10 \mathrm{~min}$, quenched to room temperature, and then unloaded from the quartz tube. The graphene layer was then transferred onto an $n$-type Si (111) substrate. For this step, the graphene/ $\mathrm{Cu}$-foil surface was coated with polymethyl methacrylate (PMMA). The PMMA/graphene was separated from the Cu-foil using a metal etchant solution. The floating and separated PMMA/graphene was then transferred by a fishing method with the $\mathrm{Si}$ (111) substrate. While this fishing method is known to leave grain boundaries and defects, ${ }^{32}$ nanometer-scale NRs are less affected by this due to their small surface area relative to that of the grains. ${ }^{18}$ Next, the graphene/Si (111) substrate was loaded into PA-MBE. Prior to its transfer to the growth chamber, the substrate was baked at $300^{\circ} \mathrm{C}$ for $2 \mathrm{~h}$ in a preparation chamber to remove dust and moisture. Once in the growth chamber, any native oxide on the substrate surface was removed by thermal and Ga-triggered cleaning. ${ }^{33}$ For the thermal cleaning, the substrate was heated up to $950^{\circ} \mathrm{C}$ for an hour and then cooled to $675^{\circ} \mathrm{C}$. Once the temperature reached $675^{\circ} \mathrm{C}$, the substrate surface was exposed to $\mathrm{Ga}$ molecular beam flux for $5 \mathrm{~s}$. The temperature was then raised again to $950^{\circ} \mathrm{C}$ for $2 \mathrm{~min}$ to completely desorb the $\mathrm{Ga}$ atoms and oxides. Finally, the substrate was cooled down to $775^{\circ} \mathrm{C}$ to grow GaN NRs. Sample growth was initiated by simultaneously exposing the substrate surface to both atomic nitrogen and Ga molecular beam flux to preserve graphene on the $\mathrm{Si}(111)$ substrate. ${ }^{34}$ The atomic nitrogen supply corresponded to a flow rate of $1.8 \mathrm{sccm}$ and a RF power of $400 \mathrm{~W}$. Prior to growth, initial bursting and the direct supply of atomic nitrogen plasma were prevented by a shutter sitting between the nitrogen plasma cell and substrate. The GaN NRs were grown on a graphene/Si (111) substrate for $60 \mathrm{~min}$ as soon as the Ga shutter was opened. ${ }^{33}$ The presence of graphene between GaN NRs and the Si (111) substrate was confirmed by both a photoluminescence (PL) map at room temperature (Fig. S1) and a cross-sectional transmission electron microscopy (XTEM) (Fig. S2). We note that the enhanced PL characteristics of the GaN NRs grown on the graphene deposited area are a result of the modified interfacial strain and NR nucleation. ${ }^{18}$

The beam equivalent pressure (BEP) of the Ga molecular beam flux was around $6 \times 10^{-7}$ Torr and the chamber pressure was kept at $1.2 \times 10^{-5}$ Torr during the process. Figures 1 (a) and 1 (b) show the $350 \mathrm{~nm}$-long undoped GaN NRs on graphene and exhibit their coalesced columnar shape with high spatial density. Each of the GaN NRs is undoped, however, showing slightly $n$-type conductivity, since GaN is not free from background impurities. ${ }^{34}$ Next, the coalesced GaN NRs on a graphene film were further fabricated into a PD. The back 

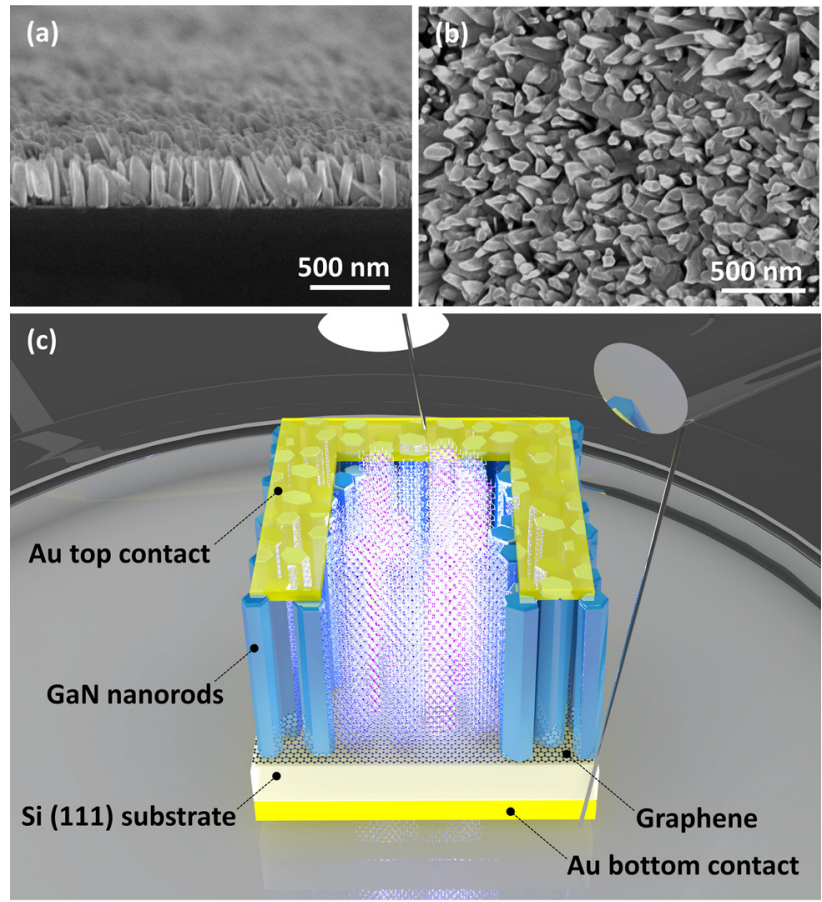

FIG. 1. SEM images for the (a) cross-sectional and (b) surface-view of GaN NRs grown on the graphene/Si (111) substrate. The GaN NRs show a coalesced columnar shape with a high spatial density. (c) Schematic illustration of a GaN NR/graphene/Si (111) heterojunction device.

and top $\mathrm{Au}(80 \mathrm{~nm})$ electrodes were deposited by electron beam evaporation to form contact pads without the planarization step. A schematic illustration of our GaN NR/graphene/Si (111) heterojunction device is presented in Fig. 1(c).

Prior to the analysis of the device's performance, the crystalline quality of coalesced GaN NRs was investigated. To ensure the crystalline quality of coalesced GaN NRs on graphene, a $10 \mathrm{~K}$ lowtemperature PL (LT-PL) measurement was performed [results shown in Fig. 2(a)]. The sample was probed with a $266 \mathrm{~nm}$ ND-YAG laser having a spot diameter of approximately $10 \mu \mathrm{m}$. During the measurement, excitation power was varied within the range of $0.03-7.5 \mathrm{~mW}$. Within that spectrum, there was an apparent donor-bound excitonic emission $\left(D^{\circ} \mathrm{X}\right)$, which indicates good crystalline quality. Also, there was a yellow luminescence peak regardless of excitation power. We are aware that well-separated self-assembled NRs have no yellow luminescence peak around $2.2 \mathrm{eV} .{ }^{35,36}$ However, we intentionally induced coalesced GaN NRs for ease of fabrication. These $\mathrm{D}^{\circ} \mathrm{X}$ and yellow luminescence peaks are strong evidence of well-coalesced GaN NRs on the graphene/Si (111) substrate that do not suffer from degraded crystalline quality.

All electrical and optical properties of the PD were measured at room temperature. The optoelectronic performance of the $\mathrm{PD}$ was tested by a Keithley 2410. A $355 \mathrm{~nm}$ UV-LED was applied as the illumination source and calibrated by a THORLABS optical power meter, as shown in Fig. 2(c). The transient response was characterized by a Yokogawa DLM2054 oscilloscope and a Stanford Research synthesized function generator (Model DS345). Figure 2(b) depicts the current-voltage $(I-V)$ curve of the GaN NR/graphene/Si (111) heterojunction in a dark condition and under the illumination of a $355 \mathrm{~nm}$ UV LED within $\pm 1 \mathrm{~V}$. The photoresponse of the heterojunction was also measured by illuminating the device with the $355 \mathrm{~nm}$ UV light with a $100 \%$ intensity of $0.78 \mathrm{~mW} / \mathrm{cm}^{2}$. The net photocurrent $I_{\text {photo }}$ of illumination can be obtained by subtracting dark current from the total current. When the device was illuminated, the current at $0.5 \mathrm{~V}$ increased from $8.57 \times 10^{-9} \mathrm{~A}$ (dark condition) to $3.0 \times 10^{-4} \mathrm{~A}$ under UV light, producing an impressively elevated current on/off ratio $\left(I_{\text {light }} / I_{\text {dark }}\right)$ of $\sim 10^{5}$, which was initiated by the GaN NRs photoconductive response. $^{37}$ This current on/off ratio was a function of the superior quality of the coalesced GaN NRs. We also obtained an open circuit potential $\left(V_{o c}\right)$ and short circuit current $\left(I_{s c}\right)$ of $0.06 \mathrm{~V}$ and $0.035 \mathrm{~mA}$, as shown in Fig. 2(d). Our GaN NR/graphene/Si (111) heterojunction device, therefore, can serve as a self-powered UV PD.

Since $\mathrm{GaN}$ has a higher absorption coefficient of about $\sim 10^{5} \mathrm{~cm}^{-1}$ in the UV range (specifically $325 \mathrm{~nm}$ ), ${ }^{38}$ this current increment is expected to be mainly initiated by the high photoconductive response of GaN NRs. To further study the interface of the GaN NR/ graphene and graphene/Si (111) substrates, transmission electron microscopy (TEM) and PL mapping across the whole wafer were obtained after the PA-MBE growth. Figure S1 shows two PL maps of intensity and full-width at half-maximum (FWHM) of the sample. These PL maps illustrate that Si (100) substrate-grown GaN NRs produced with and without graphene differ from each other. The TEM image (Fig. S2) also shows the thin graphene layer between the GaN NRs and the Si substrate. To our surprise, we found a thin layer of $\mathrm{SiO}_{2}$ between the graphene and $\mathrm{Si}$ substrate. We speculate that the thin layer of $\mathrm{SiO}_{2}$ is formed on a $\mathrm{Si}$ substrate during the graphene transfer process and it was preserved across the entire MBE growth step.

To further study the photo-excitation of the heterojunction, the $\mathrm{GaN}$ NR/graphene/Si (111) substrate was illuminated with different light intensities, ranging from $46 \mu \mathrm{W} / \mathrm{cm}^{2}$ to $0.78 \mathrm{~mW} / \mathrm{cm}^{2}$ [Fig. 3(a)]. Figure 3(a) illustrates that the current and light intensity are highly dependent on each other. Figure $3(\mathrm{~b})$ presents the photoresponse of GaN NR/graphene/Si (111) PD when illuminated with different light intensities at zero bias voltage. The result shows that, at $100 \mathrm{~Hz}$ frequency, the current alternately switched between high and low conductance with good constancy and repeatability as the UV light was turned on and off. Notably, the current increased a small amount even when irradiated with higher light intensity. Considering that the result is at a high frequency of $100 \mathrm{~Hz}$, we conclude that our GaN NR/ graphene/Si (111) PD can detect a very weak UV light, leading to a self-powered UV PD. The responsivity (R) and specific detectivity $\left(\mathrm{D}^{*}\right)$ of our coalesced GaN NR/graphene/Si (111) PD device at various light intensities are plotted in Fig. 3(c), based on the following equations:

$$
\begin{gathered}
R=\frac{I_{P}}{P_{o p t}}, \\
D^{*}=\frac{\sqrt{A} R}{\sqrt{2 e I_{d}}},
\end{gathered}
$$

where $I_{p}$ is the net photocurrent obtained (subtracting dark current from the total current), $P_{\text {opt }}$ is the incident light intensity, $A$ is the device area, and $I_{d}$ is the PD's dark current. At a $48 \mu \mathrm{W} / \mathrm{cm}^{2}$ light intensity, $\mathrm{R}$ is 17.4 A/W and $\mathrm{D}^{*}$ is $1.23 \times 10^{13}$ Jones ( 1 Jones $=1 \mathrm{~cm} \mathrm{~Hz} / 2 / \mathrm{W}$ ), 

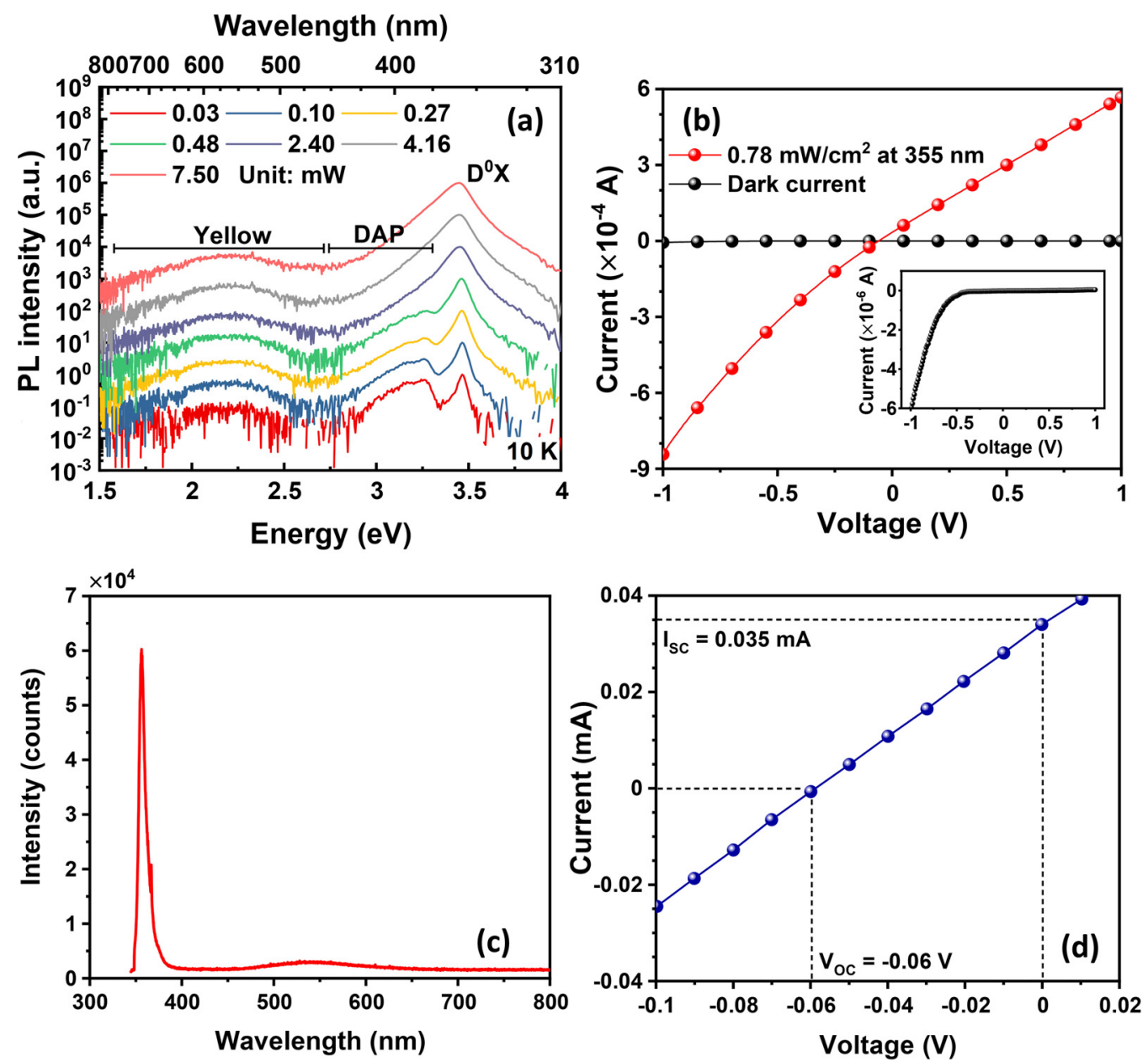

FIG. 2. (a) $10 \mathrm{~K} L T-P L$ spectra of the GaN NRs. The presence of $D^{\circ} X$ indicates high crystalline quality, and the presence of carbon shows the yellow luminescence of GaN NRs. (b) The $I-V$ curves of GaN NR/graphene/Si (111) heterojunction devices under dark conditions and under light illumination ( $355 \mathrm{~nm})$ within $\pm 1 \mathrm{~V}$ bias voltage (the inset is the magnification of the dark current). (c) Response spectra of the $355 \mathrm{~nm}$ UV LED. (d) Photovoltaic effect of the GaN/graphene heterojunction device.

which are much higher than the $\mathrm{R}$ and $\mathrm{D}^{*}$ of other PDs (see Table I). These figures also illustrate that $\mathrm{R}$ and $\mathrm{D}^{*}$ are both inversely proportional to the light intensity. The light intensity dependence of the photocurrent is plotted in Fig. 3(d) and can be described by the following power law equation:

$$
I=A P^{\alpha}
$$

where $A, P$, and $\alpha$ are constant for a given wavelength, light intensity, and photocurrent response to the light intensity. By fitting the data, $\alpha$ was determined to be 0.13 . The carrier trap states between the conduction band edge and Fermi level are believed to affect this fractional power equation.

A circuit connecting the GaN NR/graphene/Si (111) PD, function generator, and oscilloscope was tested to further explore the PD's response speed. A function generator modulated the frequency of the UV light, while the oscilloscope measured and displayed the photocurrent as a function of time. The photoresponse characteristics of the
GaN NR/graphene/Si (111) PD were measured in the frequency range of $1 \mathrm{~Hz}-20 \mathrm{kHz}$ by illumination with $355 \mathrm{~nm}$ UV light at an intensity of $0.78 \mathrm{~mW} / \mathrm{cm}^{2}$ at $0 \mathrm{~V}$ bias voltage. The result, which is presented in Figs. 4(a) and 4(b), confirms the PD's responsiveness even at a high speed of $20 \mathrm{kHz}$, indicating that it is capable of detecting a high-speed UV light signal. Two essential characterizations of the response speed are the rise time $\left(\tau_{\mathrm{r}}\right)$ and fall time $\left(\tau_{\mathrm{f}}\right)$. As shown in Fig. $4(\mathrm{a})$, at $1 \mathrm{~Hz}$ frequency, both the rise time $\left(\tau_{\mathrm{r}}\right)$ and the fall time $\left(\tau_{\mathrm{f}}\right)$ were $1 \mathrm{~ns}$ (limited by the oscilloscope resolution). A fast response speed of 13.2/13.7 $\mu$ s was also obtained at $20 \mathrm{kHz}$ [Fig. 4(b)], implying that the PD can follow quickly varied UV light signals.

The photoelectric effect of the GaN NR/graphene/Si (111) heterojunction can be understood from the energy-band diagrams at the interface. Figures 4(c) and 4(d) demonstrate the energy band diagram when the device is in dark conditions and when it is irradiated with UV light. For the Fermi energy of graphene and Si to reach equilibrium, the electrons must lower their energy by flowing to the graphene, positively charging the region near the junction. This results in 

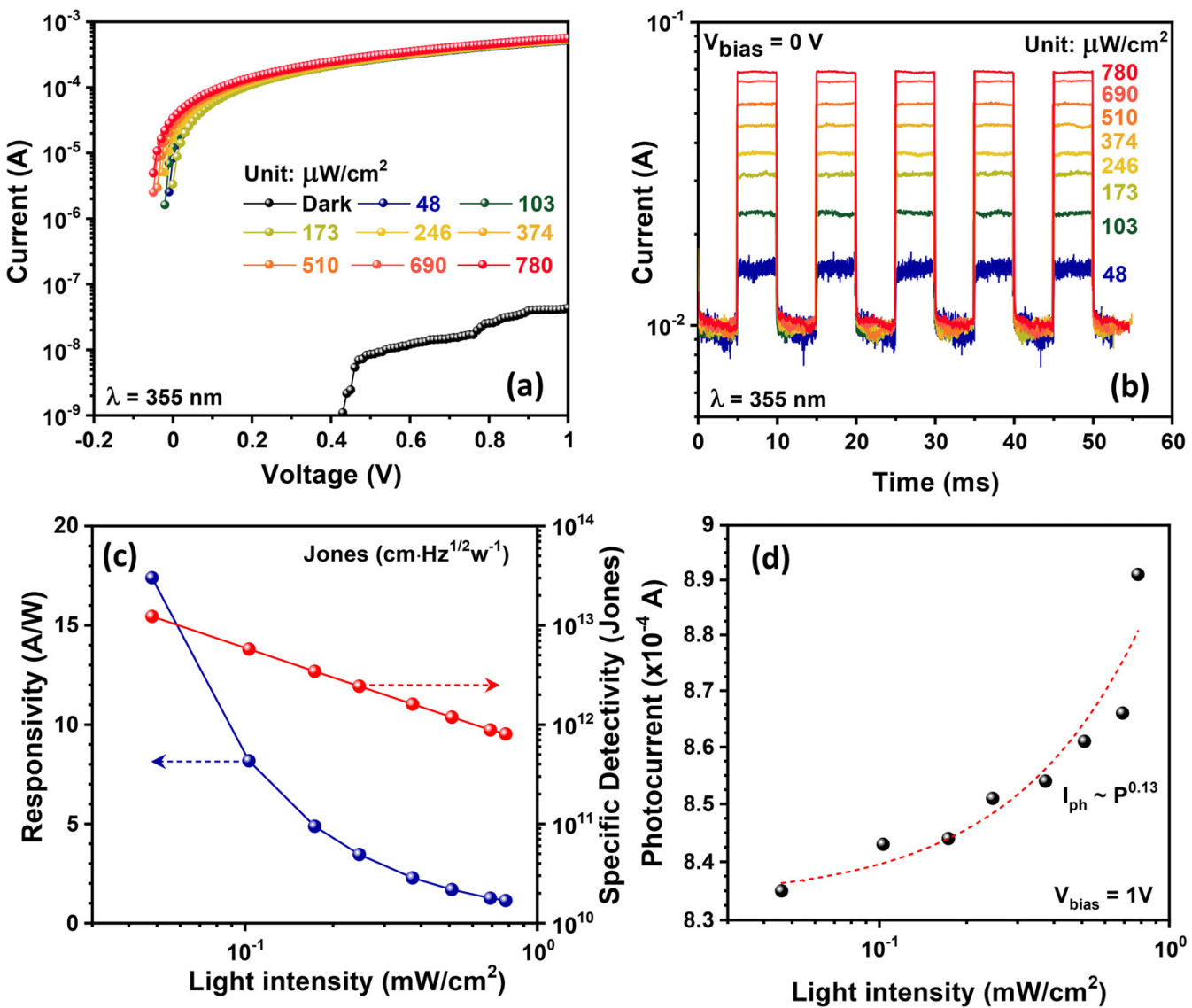

FIG. 3. (a) The $I-V$ characteristics of the junction illuminated by a $355 \mathrm{~nm}$ laser with different laser powers. $100 \%$ laser power density is $0.78 \mathrm{~mW} / \mathrm{cm}^{2}$. (b) Photoresponse of the GaN NR/graphene/Si (111) heterojunction PD by varying light intensities. (c) Light intensity-dependent responsivity and specific detectivity. (d) Logarithmic plot of the photocurrent vs light intensity.

an upward energy band bending from the Si to the graphene/Si (111) interface and triggers a built-in electric field around the junction. The difference in the work function of $\mathrm{GaN}(4.1 \mathrm{eV})$ and graphene $(4.6 \mathrm{eV})$ also causes the energy band to bend upwards, pointing from the GaN layer to the GaN NR/graphene interface and resulting in an electric field generated near the surface of the GaN NRs. Thus, at this moment (in dark conditions), electrons are gathered at the graphene layer. When the device is illuminated by incident light with photon energy

TABLE I. Comparison of the performance of GaN-based UV PDs.

\begin{tabular}{|c|c|c|c|c|c|c|}
\hline Device & $\begin{array}{l}\text { Light source, } \\
\lambda(\mathrm{nm})\end{array}$ & Self-powered & $\begin{array}{l}\text { Responsivity } \\
(\mathrm{A} / \mathrm{W}) \text { at } \mathrm{V}_{\text {bias }}\end{array}$ & $\begin{array}{l}\text { Detectivity } \\
\text { (Jones) }\end{array}$ & Response time $\left(\tau_{\mathrm{r}} / \tau_{\mathrm{f}}\right)$ & References \\
\hline GaN NR/graphene/Si (111)PD & 355 & Yes & 17.4 at $1 \mathrm{~V}$ & $1.23 \times 10^{13}$ & $\begin{array}{c}1 \mathrm{~ns} / 1 \mathrm{~ns}(1 \mathrm{~Hz}){ }^{\mathrm{a}} \\
13.2 / 13.7 \mu \mathrm{s}(20 \mathrm{kHz})\end{array}$ & This work \\
\hline GaN/Si (111) MSM PD & 362 & No & 0.183 at $15 \mathrm{~V}$ & l & I & 39 \\
\hline un-GaN with ATO electrodes PD & 325 & No & 0.095 at $5 \mathrm{~V}$ & l & l & 6 \\
\hline $\mathrm{ZnO} / \mathrm{GaN} \mathrm{PD}$ & 350 & No & 0.225 at $1 \mathrm{~V}$ & $4.83 \times 10^{13}$ & l & 40 \\
\hline Graphene/p-GaN phototransistor & 325 & Yes & 0.361 at $10 \mathrm{~V}$ & $1.5 \times 10^{10}$ & $5.05 \mathrm{~ms} / 5.11 \mathrm{~ms}$ & 27 \\
\hline GaN/graphene PD & 360 & No & 2.0 & l & l & 20 \\
\hline $\begin{array}{l}\text { Graphene/GaN NRs with graphene } \\
\text { as the contact PD }\end{array}$ & 357 & Yes & 25.0 at $1 \mathrm{~V}$ & l & I & 26 \\
\hline
\end{tabular}

${ }^{a} 1$ ns was the oscilloscope's resolution limit. 

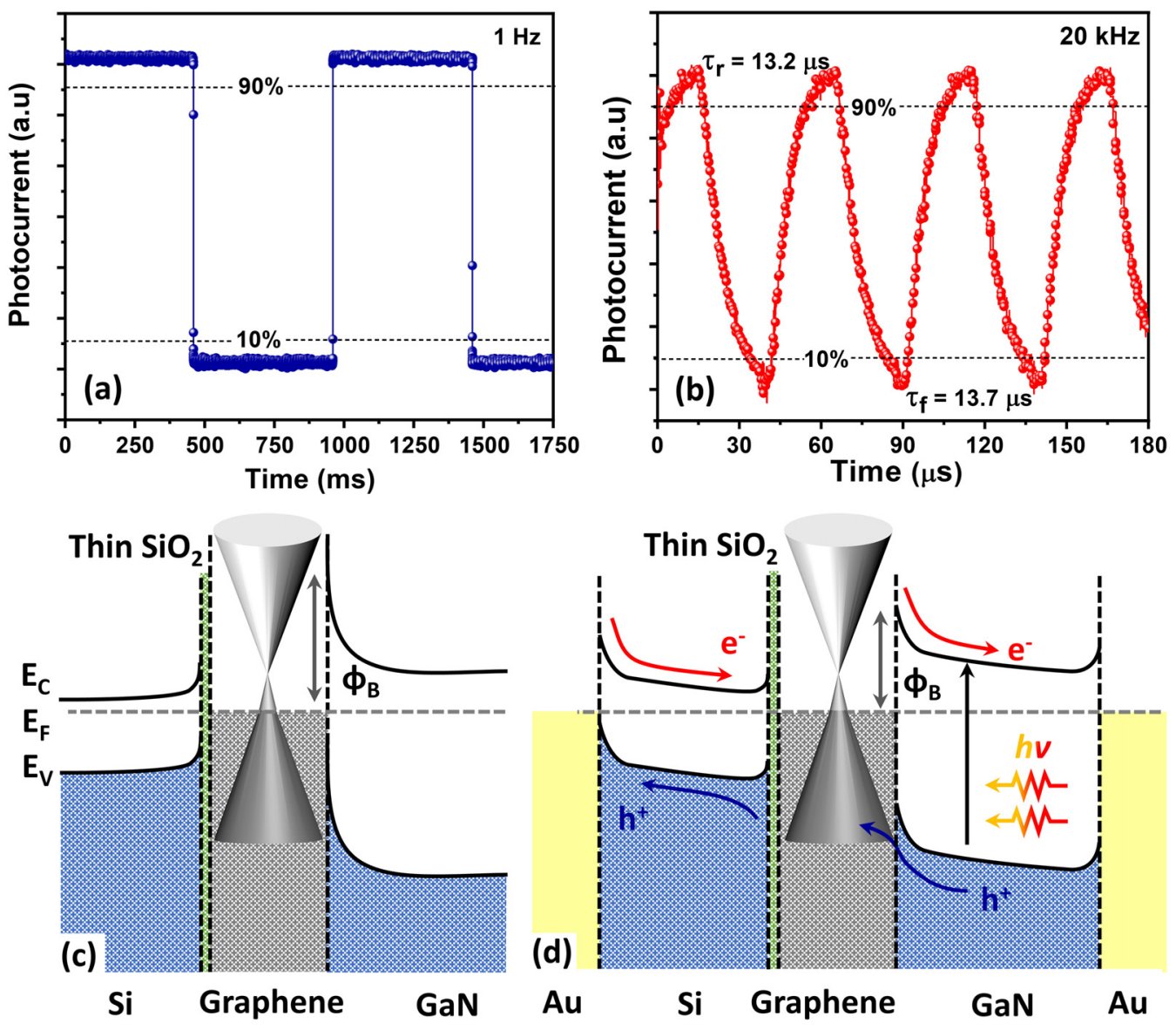

FIG. 4. Photoresponse characteristics of the GaN NR/graphene/Si (111) heterojunction to pulsed light irradiation at frequencies of (a) $1 \mathrm{~Hz}$ and (b) $20 \mathrm{kHz}$, under a voltage of $0 \mathrm{~V}$, and the estimating rise time $\left(\tau_{\mathrm{r}}\right)$ and fall time $\left(\tau_{\mathrm{f}}\right)$. Schematic band structures of the device under the (c) darkness and (d) illumination.

larger than the bandgap energy of $\mathrm{GaN}(3.4 \mathrm{eV})$, the light is absorbed by the GaN NRs, generating the electron-hole pairs. The built-in electric field at both junctions, quickly separating them, induces an effect on the recombination of the photogenerated electron-hole. The graphene sheet in the device is believed to function as a fast charge transport channel between the Ga NRs and the Si (111). Electrons are brought to the graphene and the Si more quickly than the recombination time and then collected by the electrodes to generate a large output signal. This double Schottky mechanism, fast-tunneling properties of graphene, results in higher dark current and faster switching devices. An asymmetrical metal contact consisting of two different Schottky junctions, $\mathrm{Au} / \mathrm{GaN}$ NRs and $\mathrm{Au} / \mathrm{Si}$ (111) substrate, determined the positive and negative bias of the dark current.

To summarize, a high-performance self-powered UV PD of a large-area coalesced GaN NR/graphene/Si (111) PD of $100 \mathrm{~mm}^{2}$ was investigated. Our testing revealed that the PD has spectacular photoresponse properties of $17.4 \mathrm{~A} / \mathrm{W}$, a specific detectivity of $1.23 \times 10^{13}$ Jones, and a high current on/off ratio of nearly $10^{5}$ under $355 \mathrm{~nm}$ illumination at an intensity of $48 \mu \mathrm{W} / \mathrm{cm}^{2}$. The device also responds quickly to the UV signal of $\sim 13 \mu \mathrm{s}(20 \mathrm{kHz})$ at zero bias voltage. In other words, the results of our testing reveal outstanding overall performance compared to previously reported GaN-based PDs, and our high-performing GaN NR/graphene/Si (111) PD has numerous potential applications.

See the supplementary material for characterization details of the GaN NR/graphene/Si (111) interface.

\section{AUTHORS' CONTRIBUTIONS}

N.A.A.Z. and K.P. contributed equally to this work.

The authors extend special thanks to Dr. Mohammad Khaled Shakfa and Ram Chandra Subedi of the Photonics Laboratory, King Abdullah University of Science and Technology, Saudi Arabia, for their useful discussions and PL measurement. The authors declare that they have no competing financial interest. R. Zakaria and N. A. A. Zulkifli would like to acknowledge support from the University of Malaya (No. IIRG03-2019). K. Park would like to acknowledge support from the National Research Foundation of Korea (NRF) grant funded by the Korea Government (MSIT) (No. NRF2020R1F1A1070471). 


\section{DATA AVAILABILITY}

The data that support the findings of this study are available from the corresponding author upon reasonable request.

\section{REFERENCES}

${ }^{1}$ A. R. Acharya, Himalayan Phys. 5, 22 (2015).

${ }^{2}$ P. Gupta, A. A. Rahman, S. Subramanian, S. Gupta, S. Thamizhavel, T. Orlova, S. Rouvimov, S. Vishwanath, V. Protasenko, and M. R. Laskar, Sci. Rep. 6, 23708 (2016).

${ }^{3}$ H. Sekiguchi, K. Kato, J. Tanaka, A. Kikuchi, and K. Kishino, Phys. Status Solidi 205, 1067 (2008).

${ }^{4}$ S. Guha and N. A. Bojarczuk, Appl, Phys. Lett. 72, 415 (1998).

${ }^{5}$ T.-L. Wu, D. Marcon, S. You, N. Posthuma, B. Bakeroot, S. Stoffels, M. Van Hove, G. Groeseneken, and S. Decoutere, IEEE Electron Device Lett. 36, 1001 (2015).

${ }^{6}$ M. L. Tu, Y. K. Su, S. J. Chang, and R. W. Chuang, J. Cryst. Growth 298, 744 (2007).

${ }^{7}$ C. Zhao, T. K. Ng, C. C. Tseng, J. Li, Y. Shi, N. Wei, D. Zhang, G. B. Consiglio, A. Prabaswara, A. A. Alhamoud, and A. M. Albadri, RSC Adv. 7, 26665 (2017).

${ }^{8}$ S. Chen, K. Shehzad, X. Qiao, X. Luo, X. Liu, Y. Zhang, X. Zhang, Y. Xu, and X. Fan, J. Mater. Chem. C 6, 11078 (2018).

${ }^{9}$ D. Liu, H. J. Li, J. Gao, S. Zhao, Y. Zhu, P. Wang, D. Wang, A. Chen, X. Wang, and J. Yang, Nanoscale Res. Lett. 13, 261 (2018).

${ }^{10} \mathrm{X}$. Zhang, Q. Liu, B. Liu, W. Yang, J. Li, P. Niu, and X. Jiang, J. Mater. Chem. C 5, 4319 (2017).

${ }^{11}$ C. Zhao, N. Alfaraj, R. C. Subedi, J. W. Liang, A. A. Alatawi, A. A. Alhamoud, M. Ebaid, M. S. Alias, T. K. Ng, and B. S. Ooi, Prog. Quantum Electron. 61, 1 (2018).

${ }^{12}$ C. Soci, A. Zhang, B. Xiang, S. A. Dayeh, D. P. R. Aplin, J. Park, X. Y. Bao, Y. H. Lo, and D. Wang, Nano Lett. 7, 1003 (2007).

${ }^{13}$ Y. Zou, Y. Zhang, Y. Hu, and H. Gu, Sensors 18, 2072 (2018).

${ }^{14}$ W. Guo, M. Zhang, A. Banerjee, and P. Bhattacharya, Nano Lett. 10, 3355 (2010).

${ }^{15}$ R. Calarco, R. J. Meijers, R. K. Debnath, T. Stoica, E. Sutter, and H. Lüth, Nano Lett. 7, 2248 (2007).

${ }^{16}$ S. D. Hersee, X. Sun, and X. Wang, Nano Lett. 6, 1808 (2006).

${ }^{17}$ G. T. Wang, A. A. Talin, D. J. Werder, J. R. Creighton, E. Lai, R. J. Anderson, and I. Arslan, Nanotechnology 17, 5773 (2006).

${ }^{18}$ V. Kumaresan, L. Largeau, A. Madouri, F. Glas, H. Zhang, F. Oehler, A. Cavanna, A. Babichev, L. Travers, N. Gogneau, and M. Tchernycheva, Nano Lett. 16, 4895 (2016).
${ }^{19}$ T. Journot, V. Bouchiat, B. Gayral, J. Dijon, and B. Hyot, ACS Appl. Mater. Interfaces 10, 18857 (2018).

${ }^{20}$ D. Periyanagounder, G. Paulraj, V. Purushothaman, H. Jr-Hau, and K. Jeganathan, J. Mater. Chem. C 6, 9545 (2018).

${ }^{21}$ K. I. Bolotin, K. J. Sikes, Z. Jiang, M. Klima, G. Fudenberg, J. Hone, P. Kim, and H. L. Stormer, Solid State Commun. 146, 351 (2008).

${ }^{22}$ W. Wang, Y. Xiao, X. Li, Q. Cheng, and G. Wang, Chem. Eng. J. 371, 327 (2019).

${ }^{23}$ C. Xie, Y. Wang, Z. X. Zhang, D. Wang, and L. B. Luo, Nano Today 19, 41 (2018).

${ }^{24}$ Z. Zulkifli, S. M. Shinde, T. Suguira, G. Kalita, and M. Tanemura, Appl. Surf. Sci. 356, 674 (2015).

${ }^{25}$ J. Sun, M. T. Cole, N. Lindvall, K. B. Teo, and A. Yurgens, Appl. Phys. Lett. 100, 022102 (2012).

${ }^{26}$ A. V. Babichev, H. Zhang, P. Lavenus, F. H. Julien, A. Y. Egorov, Y. T. Lin, L. W. Tu, and M. Tchernycheva, Appl. Phys. Lett. 103, 201103 (2013).

${ }^{27}$ H. Tian, Q. Liu, A. Hu, X. He, Z. Hu, and X. Guo, Opt. Express 26, 5408 (2018).

${ }^{28}$ R. K. Debnath, R. Meijers, T. Richter, T. Stoica, R. Calarco, and H. Lüth, Appl. Phys. Lett. 90, 123117 (2007).

${ }^{29}$ H. P. T. Nguyen, K. Cui, S. Zhang, M. Djavid, A. Korinek, G. A. Botton, and Z. Mi, Nano Lett. 12, 1317 (2012).

${ }^{30}$ S. Perraud, S. Poncet, S. Noël, M. Levis, P. Faucherand, E. Rouvière, P. Thony, C. Jaussaud, and R. Delsol, Solar Energy Mater. Solar Cells 93, 1568 (2009).

${ }^{31}$ K. Park, J. W. Min, R. C. Subedi, M. K. Shakfa, B. Davaasuren, T. K. Ng, B. S. Ooi, C. Kang, and J. Kim, Appl. Surf. Sci. 522, 146422 (2020).

${ }^{32}$ D. Kireev, D. Sarik, T. Wu, X. Xie, B. Wolfrum, and A. Offenhäusser, Carbon 107, 319 (2016).

${ }^{33}$ J. H. Lee, Z. M. Wang, and G. J. Salamo, Appl. Phys. Lett. 88, 252108 (2006).

${ }^{34}$ I. M. Høiaas, A. Liudi Mulyo, P. E. Vullum, D.-C. Kim, L. Ahtapodov, B.-O. Fimland, K. Kishino, and H. Weman, Nano Lett. 19, 1649 (2019).

${ }^{35}$ A. L. Mulyo, Y. Konno, J. S. Nilsen, A. T. J. van Helvoort, B.-O. Fimland, H. Weman, and K. Kishino, J. Cryst. Growth 480, 67 (2017).

${ }^{36}$ I. M. Tiginyanu, V. V. Ursaki, V. V. Zalamai, S. Langa, S. Hubbard, D. Pavlidis, and H. Föll, Appl. Phys. Lett. 83, 1551 (2003).

${ }^{37}$ R. Calarco, M. Marso, T. Richter, A. I. Aykanat, R. Meijers, A. vd Hart, T. Stoica, and H. Lüth, Nano Lett. 5, 981 (2005).

${ }^{38} \mathrm{G}$. Yu, G. Wang, H. Ishikawa, M. Umeno, T. Soga, T. Egawa, J. Watanabe, and T. Jimbo, Appl. Phys. Lett. 70, 3209 (1997).

${ }^{39}$ L. Ravikiran, K. Radhakrishnan, N. Dharmarasu, M. Agrawal, Z. Wang, A. Bruno, C. Soci, T. Lihuang, and K. S. Ang, IEEE Sens. J. 17, 72 (2017).

${ }^{40}$ M. Mishra, A. Gundimeda, T. Garg, A. Dash, S. Das, and G. Gupta, Appl. Surf. Sci. 478, 1081 (2019) 